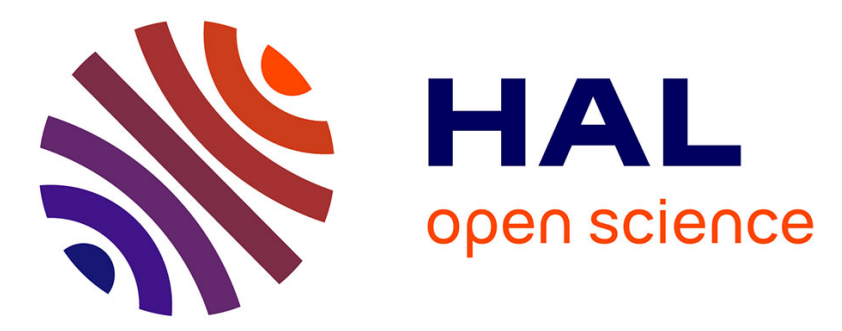

\title{
Maximum a Posteriori Consistent Estimation Using Interval Analysis
}

Manel Abid, Michel Kieffer, Beatrice Pesquet-Popescu

\section{To cite this version:}

Manel Abid, Michel Kieffer, Beatrice Pesquet-Popescu. Maximum a Posteriori Consistent Estimation Using Interval Analysis. SYSID 2012, Jul 2012, Bruxelles, Belgium. pp.1-6. hal-00727521

\section{HAL Id: hal-00727521 \\ https://hal-centralesupelec.archives-ouvertes.fr/hal-00727521}

Submitted on 4 Sep 2012

HAL is a multi-disciplinary open access archive for the deposit and dissemination of scientific research documents, whether they are published or not. The documents may come from teaching and research institutions in France or abroad, or from public or private research centers.
L'archive ouverte pluridisciplinaire HAL, est destinée au dépôt et à la diffusion de documents scientifiques de niveau recherche, publiés ou non, émanant des établissements d'enseignement et de recherche français ou étrangers, des laboratoires publics ou privés. 


\title{
MAXIMUM A POSTERIORI CONSISTENT ESTIMATION USING INTERVAL ANALYSIS
}

\author{
M. Abid ${ }^{*}$ M. Kieffer ${ }^{*, * *}$ B. Pesquet-Popescu* \\ * Telecom ParisTech, Signal and Image Processing Department, \\ 46 rue Barrault, 75634 Paris cedex 13, France \\ (e-mail: \{abid,pesquet\}@telecom-paristech.fr) \\ ** on leave from L2S - CNRS - SUPELEC - Univ Paris-Sud, \\ 91192 Gif-sur-Yvette, France (e-mail: kieffer@lss.supelec.fr)
}

\begin{abstract}
This paper presents a MAP estimator for some vector $\mathbf{x}$ from its quantized and noisy linear measurements. The complexity of the optimal MAP estimator is intractable in general. Two suboptimal solutions have been proposed, one of which being iterative to be able to handle large-scale problems. Leveraging on techniques from interval analysis, it is possible to quickly eliminate solutions which are not consistent with the signal model, and the quantization noise. These techniques have been applied to the estimation of the input signal of an OFB using noisy measurements of its quantized subbands. The experimental results show that when the channel is noisy, this approach performs better in terms of reconstruction SNR than classical least-squares reconstruction.
\end{abstract}

\section{INTRODUCTION}

This paper considers maximum a posteriori (MAP) estimation of the realization $\mathbf{x}$ of some random (correlated) vector $\mathbf{X}$ from the noisy observations $\mathbf{r}$ of quantized linear measurements $\mathbf{y}=\mathbf{H x}$, where $\mathbf{H}$ is an $M \times N$ matrix. This estimation problem known as a linear mixing problem has been already handled using belief propagation to evaluate the marginal posterior probability distribution of each entry of the vector $\mathbf{x}$ knowing $\mathbf{r}$, see, e.g., Rangan [2010, 2011]. An implementation of this technique based on message passing (MP) algorithms on a graph Kschischang et al. [2001], Donoho et al. [2009] is very complex as it involves high-dimensional integration. This issue is addressed using Gaussian approximations Rangan [2011]. Nevertheless, correlation in the components of $\mathbf{x}$ is difficult to take into account as it leads to many cycles in the graph representing the relation between variables, which is detrimental to MP algorithms.

In the particular case of an expansion, i.e., when $M>N$, the linear dependencies between the samples of $\mathbf{y}$ can be exploited jointly with the bounded quantization noise to perform a consistent MAP estimation with a reasonable complexity. This paper shows that this problem can be formulated as a constrained optimization problem to which a suboptimal solution can be delivered by solving several linear programs or by using tools from interval analysis Jaulin et al. [2001].

Schemes operating such signal expansion are for example oversampled filter banks (OFB) Vaidyanathan [1993]. The output subbands generated by the OFB provide an overcomplete representation of the signal placed at its input.

\footnotetext{
* This work was partly supported the ANR CPP project. Michel Kieffer is partly supported by the Institut Universitaire de France.
}

The quantized subbands are usually transmitted through a noisy or lossy channel. The redundancy introduced in the subbands can then be used at the receiver side to correct the transmission errors, see, e.g., Redinbo [2000], Labeau et al. [2005], Abid et al. [2011] or to compensate the erasures, see, e.g., Goyal et al. [2001], Kovacević et al. [2002], Rath and Guillemot [2004], Akbari and Labeau [2010].

This paper presents the MAP estimator for the linear mixing problem. Two suboptimal implementations of the MAP estimator are introduced. A first implementation is suitable for small-size input vectors. A second iterative suboptimal implementation is able to perform the estimation of large input signals with a manageable complexity. This algorithm is well-suited to the estimation of the input of an OFB from its noisy output.

The rest of the paper is organized as follows. The estimation problem is presented in Section 2. The formulation of the optimal MAP estimator is developed in Section 3. Suboptimal implementations are detailed in Section 4. Finally two examples are presented in Sections 5 and 6 before providing some conclusions.

\section{PROBLEM STATEMENT}

Assume that some random vector $\mathbf{X} \in \mathbb{R}^{N}$ has to be transmitted from a source through a channel to a receiver, see Figure 1. The probability density function (pdf) of $\mathbf{X}$ is $p_{\mathbf{X}}(\mathbf{x})$. Prior to transmission, an expansion of $\mathbf{X}$ is performed using an $M \times N$ matrix $\mathbf{H}$ with $M>N$ of rank $N$ to get $\mathbf{Y} \in \mathbb{R}^{M}$ with

$$
\mathbf{Y}=\mathbf{H X},
$$

see Goyal [2002]. Each component $Y_{m}$ of $\mathbf{Y}$ is then quantized with a scalar quantizer $q$ of rate $\rho$. The quantization 
intervals $\left[s_{0}\right] \ldots\left[s_{2^{\rho}-1}\right]$ form a partition of $\mathbb{R}$. At quantizer output, one gets a random vector $\mathbf{Z}$ of quantization indexes, with

$$
Z_{m}=q\left(Y_{m}\right)=z \Leftrightarrow Y_{m} \in\left[s_{z}\right], z \in\left\{0 \ldots 2^{\rho}-1\right\} .
$$

These indexes are then transmitted through some noisy channel, assumed to be memoryless. This channel represents the binarization, channel coding and modulation steps as well as the physical channel and the corresponding demodulation and channel decoding steps Anderson [2005]. The memoryless channel assumption holds provided that proper interleaving is performed, e.g., prior to channel coding. The channel output related to $Z_{m}$ is assumed to be described by a real or complex random vector $\mathbf{R}_{m}, m=1 \ldots M$. The effect of the channel is then described by the channel transition probability $p_{R \mid Z}(\mathbf{r} \mid z)$.

The aim of this paper is to evaluate the MAP estimate of the realization $\mathbf{x}$ of $\mathbf{X}$ from the observation of $\mathbf{r}_{m}$, $m=1 \ldots M$ at channel output,

$$
\widehat{\mathbf{x}}_{\mathrm{MAP}}=\arg \max _{\mathbf{x}} p_{X \mid R}\left(\mathbf{x} \mid \mathbf{r}_{1: M}\right),
$$

where for a set of vectors $\mathbf{a}_{1} \ldots \mathbf{a}_{k}, \mathbf{a}_{1: k}^{T}=\left(\mathbf{a}_{1}^{T} \ldots \mathbf{a}_{k}^{T}\right)$.

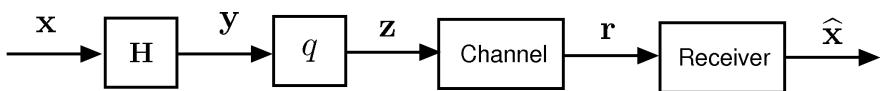

Fig. 1. Considered transmission scheme

\section{MAP ESTIMATOR}

Using Bayes' rule, one may rewrite (3) as

$$
\widehat{\mathbf{x}}_{\mathrm{MAP}}=\arg \max _{\mathbf{x}} p_{R \mid X}\left(\mathbf{r}_{1: M} \mid \mathbf{x}\right) p_{X}(\mathbf{x})
$$

where

$$
\begin{aligned}
p_{R \mid X}\left(\mathbf{r}_{1: M} \mid \mathbf{x}\right) & =\sum_{\mathbf{z}_{1: M}} p_{R Z \mid X}\left(\mathbf{r}_{1: M}, \mathbf{z}_{1: M} \mid \mathbf{x}\right) \\
& =\sum_{\mathbf{z}_{1: M}} p_{R \mid Z X}\left(\mathbf{r}_{1: M} \mid \mathbf{z}_{1: M}, \mathbf{x}\right) p_{Z \mid X}\left(\mathbf{z}_{1: M} \mid \mathbf{x}\right) .
\end{aligned}
$$

In (5), once $\mathbf{z}_{1: M}$ is known, $\mathbf{x}$ does not bring additional knowledge on $\mathbf{r}_{1: M}$, and since the channel is memoryless, one gets

$$
p_{R \mid Z X}\left(\mathbf{r}_{1: M} \mid \mathbf{z}_{1: M}, \mathbf{x}\right)=\prod_{m=1}^{M} p_{R \mid Z}\left(\mathbf{r}_{m} \mid z_{m}\right) .
$$

Moreover, since a scalar quantization has been considered, one has

$$
p_{Z \mid X}\left(\mathbf{z}_{1: M} \mid \mathbf{x}\right)=p_{Z \mid X}\left(\mathbf{z}_{1: M} \mid \mathbf{H} \mathbf{x}\right)=\prod_{m=1}^{M} p_{Z \mid X}\left(z_{m} \mid \mathbf{h}_{m}^{T} \mathbf{x}\right)
$$

where $\mathbf{h}_{m}^{T}$ is the $m$-th row of $\mathbf{H}$. According to (2), one has

$$
p_{Z \mid X}\left(z_{m} \mid \mathbf{h}_{m}^{T} \mathbf{x}\right)=I_{\left[s_{z_{m}}\right]}\left(\mathbf{h}_{m}^{T} \mathbf{x}\right),
$$

where the indicator function is

$$
I_{[s]}(x)= \begin{cases}1 & \text { if } x \in[s] \\ 0 & \text { else. }\end{cases}
$$

Finally, combining (5)-(9) in (4), one obtains

$$
\widehat{\mathbf{x}}_{\mathrm{MAP}}=\arg \max _{\mathbf{x}} p_{X}(\mathbf{x}) \sum_{\mathbf{z}_{1: M}} \prod_{m=1}^{M} p_{R \mid Z}\left(\mathbf{r}_{m} \mid z_{m}\right) I_{\left[s_{z_{m}}\right]}\left(\mathbf{h}_{m}^{T} \mathbf{x}\right) \text {. }
$$

For a given $\mathbf{x}$, by definition of the indicator function and due to the fact that the quantization intervals form a partition of $\mathbb{R}$, the sum over $\mathbf{z}_{1: M}$ consists of a single nonzero term when $z_{m}=q\left(\mathbf{h}_{m}^{\prime} \mathbf{x}\right), m=1 \ldots M$. Thus $(10)$ becomes

$$
\widehat{\mathbf{x}}_{\mathrm{MAP}}=\arg \max _{\mathbf{x}} p_{X}(\mathbf{x}) \prod_{m=1}^{M} p_{R \mid Z}\left(\mathbf{r}_{m} \mid q\left(\mathbf{h}_{m}^{T} \mathbf{x}\right)\right) .
$$

Obtaining $\widehat{\mathbf{x}}_{\mathrm{MAP}}$ requires the maximization of

$$
f\left(\mathbf{x}, \mathbf{r}_{1: M}\right)=p_{X}(\mathbf{x}) \prod_{m=1}^{M} p_{R \mid Z}\left(\mathbf{r}_{m} \mid q\left(\mathbf{h}_{m}^{T} \mathbf{x}\right)\right)
$$

over all possible $\mathbf{x}$. The evaluation of $f\left(\mathbf{x}, \mathbf{r}_{1: M}\right)$ is easy, however, due to the quantization, this function is only piecewise-continuous, which makes its maximization very difficult, especially when $N$ is large.

\section{SUBOPTIMAL IMPLEMENTATIONS}

\subsection{Negligible channel noise}

Assume first that the noise introduced by the channel is very small. It is likely that $p_{R \mid Z}\left(\mathbf{r}_{m} \mid z_{m}\right)$ will be vanishing for all values of $z_{m}$, except for some $z_{m}^{*} \in\left\{0 \ldots 2^{\rho}-1\right\}$, which is very likely to correspond to the actual transmitted quantization index $q\left(y_{m}\right)$. In this case, when considering in (10) the sum over $\mathbf{z}_{1: M}$, only the term corresponding to $\mathbf{z}_{1: M}^{*}$ may be kept and one gets

$$
\widehat{\mathbf{x}}_{\mathrm{MAP}}=\arg \max _{\mathbf{x}} p_{X}(\mathbf{x}) \prod_{m=1}^{M} p_{R \mid Z}\left(\mathbf{r}_{m} \mid z_{m}^{*}\right) I_{\left[s_{z_{m}^{*}}\right]}\left(\mathbf{h}_{m}^{T} \mathbf{x}\right) .
$$

Now, $\prod_{m=1}^{M} p_{R \mid Z}\left(\mathbf{r}_{m} \mid z_{m}^{*}\right)$ does not depend any more of $\mathbf{x}$ and (13) may be written as a constrained optimization problem

$$
\begin{aligned}
& \widehat{\mathbf{x}}_{\mathrm{MAP}}=\arg \max _{\mathbf{x}} p_{X}(\mathbf{x}) \\
& \quad \text { st } \mathbf{h}_{m}^{T} \mathbf{x} \in\left[s_{z_{m}^{*}}\right], m=1 \ldots M,
\end{aligned}
$$

which may be written with linear constraints as follows

$$
\begin{gathered}
\widehat{\mathbf{x}}_{\mathrm{MAP}}=\arg \max _{\mathbf{x}} p_{X}(\mathbf{x}) \\
\text { st } \mathbf{H} \mathbf{x} \leqslant \overline{\mathbf{s}}\left(\mathbf{z}_{1: M}^{*}\right), \\
-\mathbf{H x} \leqslant-\underline{\mathbf{s}}\left(\mathbf{z}_{1: M}^{*}\right),
\end{gathered}
$$

where $\overline{\mathbf{s}}\left(\mathbf{z}_{1: M}\right)=\left(\bar{s}_{z_{1}} \ldots \bar{s}_{z_{M}}\right)^{T}$ and $\underline{\mathbf{s}}\left(\mathbf{z}_{1: M}\right)=\left(\underline{s}_{z_{1}} \ldots \underline{s}_{z_{M}}\right)^{T}$ with $\bar{s}_{z_{m}}$ and $\underline{s}_{z_{m}}$ the upper and lower bounds of the interval $\left[s_{z_{m}}\right]$.

\subsection{General case}

Now assume that the noise introduced by the channel is no more negligible. The vector $\mathbf{z}_{1: M}^{*}$ maximizing $p_{R \mid Z}\left(\mathbf{r}_{1: M} \mid \mathbf{z}_{1: M}\right)$ may not necessarily correspond to the vector of transmitted indexes $q\left(\mathbf{y}_{1: M}\right)$. It may even not correspond to a vector of quantization indexes that may be obtained from a given $\mathbf{H x}$, due to the relation between the entries of $\mathbf{H x}$. The idea of the proposed suboptimal implementation of (3) is then to keep in (10) in the sum over $\mathbf{z}_{1: M}$ only the vector $\mathbf{z}_{1: M}^{\mathrm{f}}$ maximizing $p_{R \mid Z}\left(\mathbf{r}_{1: M} \mid \mathbf{z}_{1: M}\right)$ and such that there exists some feasible $\mathbf{x} \in \mathbb{R}^{N}$, i.e., some $\mathbf{x}$ satisfying $q(\mathbf{H x})=\mathbf{z}_{1: M}^{\mathrm{f}}$. Then (15) is solved with $\mathbf{z}_{1: M}^{\mathrm{f}} \cdot$ 
Assume that the values of $\mathbf{z}_{1: M}$ are sorted in decreasing value of $p_{R \mid Z}\left(\mathbf{r}_{1: M} \mid \mathbf{z}_{1: M}\right)$ to get $\left(\mathbf{z}_{1: M}^{(1)} \ldots \mathbf{z}_{1: M}^{(L)}\right)$, i.e., $\ell_{1}<$ $\ell_{2}$ implies

$$
p_{R \mid Z}\left(\mathbf{r}_{1: M} \mid \mathbf{z}_{1: M}^{\left(\ell_{1}\right)}\right) \geqslant p_{R \mid Z}\left(\mathbf{r}_{1: M} \mid \mathbf{z}_{1: M}^{\left(\ell_{2}\right)}\right),
$$

with $L=2^{\rho M}$. To determine whether there exists some $\mathbf{x} \in \mathbb{R}^{N}$ satisfying $q(\mathbf{H x})=z_{1: M}^{(\ell)}$, one may, e.g., solve the following linear program

$$
\begin{aligned}
\max _{\mathbf{x}} & \mathbf{a}^{T} \mathbf{x} \\
\text { st } & \mathbf{H} \mathbf{x} \leqslant \overline{\mathbf{s}}\left(\mathbf{z}_{1: M}^{(\ell)}\right), \\
& -\mathbf{H} \mathbf{x} \leqslant-\underline{\mathbf{s}}\left(\mathbf{z}_{1: M}^{(\ell)}\right),
\end{aligned}
$$

with increasing $\ell$ until a feasible solution is found, a being any non-zero vector. One could try to directly solve (15) with $\mathbf{z}_{1: M}^{(\ell)}$, starting from $\ell=1$ and increasing $\ell$ until a solution exists. Nevertheless, solving first (17) for various $\mathbf{z}_{1: M}^{(\ell)}$ with increasing $\ell$ is much less complex than solving (15), especially if $p_{X}(\mathbf{x})$ is a general pdf.

A suboptimal test to determine whether (17) admits a feasible $\mathbf{x}$ involves the following property. Since $\mathbf{H}$ is of rank $N$, there exists an $(M-N) \times M$ matrix $\mathbf{P}$ of rank $(M-N)$ such that

$$
\mathbf{P H x}=\mathbf{0} \text { for all } \mathbf{x} \in \mathbb{R}^{N} \text {. }
$$

In what follows, $\mathbf{P}$ is called a parity-check matrix for $\mathbf{H}$. The matrix $\mathbf{H}$ can be seen as a generator matrix for a realvalued channel code Redinbo [2000]. If $\mathbf{P y}=\mathbf{0}$ for some $\mathbf{y} \in \mathbb{R}^{M}$, there exists some $\mathbf{x} \in \mathbb{R}^{N}$ such that $\mathbf{y}=\mathbf{H x}$.

Consider the box $\left[\mathbf{s}\left(\mathbf{z}_{1: M}^{(\ell)}\right)\right]=\left(\left[s_{z_{1}^{(\ell)}}\right] \ldots\left[s_{z_{M}^{(\ell)}}\right]\right)^{T}$. The box $\mathbf{P}\left[\mathbf{s}\left(\mathbf{z}_{1: M}^{(\ell)}\right)\right]$ that can be evaluated using basic interval additions and multiplications Jaulin et al. [2001] is such that

$$
\left\{\mathbf{P y} \text { with } \mathbf{y} \in\left[\mathbf{s}\left(\mathbf{z}_{1: M}^{(\ell)}\right)\right]\right\} \subset \mathbf{P}\left[\mathbf{s}\left(\mathbf{z}_{1: M}^{(\ell)}\right)\right] .
$$

Thus,

$$
\mathbf{0} \notin \mathbf{P}\left[\mathbf{s}\left(\mathbf{z}_{1: M}^{(\ell)}\right)\right] \Rightarrow \mathbf{0} \notin\left\{\mathbf{P y} \text { with } \mathbf{y} \in\left[\mathbf{s}\left(\mathbf{z}_{1: M}^{(\ell)}\right)\right]\right\}
$$

and if $\mathbf{0} \notin \mathbf{P}\left[\mathbf{s}\left(\mathbf{z}_{1: M}^{(\ell)}\right)\right]$, then there is no solution to (17) for $\mathbf{z}_{1: M}^{(\ell)}$. The inconsistency test $\mathbf{0} \notin \mathbf{P}\left[\mathbf{s}\left(\mathbf{z}_{1: M}^{(\ell)}\right)\right]$ has a complexity between $O(M)$ and $O(M(M-N))$, which is in general lower than solving directly (17).

\subsection{Practical implementation}

The number of possible values of $\mathbf{z}_{1: M}$ to sort in Section 4.2 is $2^{\rho M}$, which may be intractable even for moderate values of $M$. For $m=1 \ldots M$, one may sort the possible values of $z_{m}$ in decreasing order of $p_{R \mid Z}\left(\mathbf{r}_{m} \mid z_{m}\right)$. One then keeps only the $N_{m}$ values of $z_{m}$ associated to the largest $p_{R \mid Z}\left(\mathbf{r}_{m} \mid z_{m}\right)$. This leads to $\prod_{m=1}^{M} N_{m}$ possible combinations of values for $\mathbf{z}_{1: M}$, which may then be sorted in decreasing order of $p_{R \mid Z}\left(\mathbf{r}_{1: M} \mid \mathbf{z}_{1: M}\right)$. Again, only the best $N^{\prime}$ vectors $\mathbf{z}_{1: M}^{(0)} \cdots \mathbf{z}_{1: M}^{\left(N^{\prime}-1\right)}$ may be kept. The procedure described in Section 4.2 may then be applied to these $N^{\prime}$ vectors.

The choice of $N_{m}$ and $N^{\prime}$ depends on the level of the channel noise, of the relative values of $p_{R \mid Z}\left(\mathbf{r}_{m} \mid z_{m}\right)$, and of the targeted complexity-efficiency trade-off.

\subsection{Iterative implementation}

When $N$ and $M$ are large, even the practical implementation of Section 4.3 may be difficult to put at work due to the number of $\mathbf{z}_{1: M}$ that have to be considered. A recursive implementation of (3) is considered here, assuming that $\mathbf{H}$ has the following form

$$
\mathbf{H}=\left(\begin{array}{ccccccc}
\mathbf{H}_{0} & \mathbf{0} & \cdots & & & \cdots & \mathbf{0} \\
\mathbf{H}_{1} & \mathbf{H}_{0} & \mathbf{0} & & & & \vdots \\
\vdots & \ddots & \ddots & \ddots & & & \\
\mathbf{H}_{K-1} & & \ddots & \ddots & \ddots & & \\
\mathbf{0} & \ddots & & \ddots & \ddots & \mathbf{0} & \vdots \\
\vdots & \ddots & \mathbf{H}_{K-1} & & \mathbf{H}_{1} & \mathbf{H}_{0} & \mathbf{0} \\
\mathbf{0} & \cdots & \mathbf{0} & \mathbf{H}_{K-1} & \cdots & \mathbf{H}_{1} & \mathbf{H}_{0}
\end{array}\right) .
$$

Such structure may be found, e.g., when considering finite impulse response filter banks Vaidyanathan [1993].

In (21), for $k=0 \ldots K-1, \mathbf{H}_{k}$ is an $M \times N$ matrix with $M>N$ and $\mathbf{H}$ is assumed to be of full rank. One may write (1), where now $\mathbf{X}=\left(\left(\mathbf{X}^{1}\right)^{T} \ldots\left(\mathbf{X}^{V}\right)^{T}\right)^{T} \in \mathbb{R}^{V N}$ and $\mathbf{Y}=\left(\left(\mathbf{Y}^{1}\right)^{T} \ldots\left(\mathbf{Y}^{V}\right)^{T}\right)^{T} \in \mathbb{R}^{V M}$, with $V$ some positive integer. Using (21), one obtains

$$
\mathbf{Y}^{v}=\sum_{u=0}^{K-1} \mathbf{H}_{u} \mathbf{X}^{v-u}, v=1 \ldots V,
$$

with $\mathbf{X}^{v}=\mathbf{0}$ if $v \leqslant 0$.

The aim is again to estimate the source outcome $\mathbf{x}$ from some channel output vector $\mathbf{r}=\left(\left(\mathbf{r}^{1}\right)^{T} \ldots\left(\mathbf{r}^{V}\right)^{T}\right)^{T}$, when $\mathbf{H}$ is described by (21). An iterative algorithm may be considered to get an approximate solution to (3). Assume, at iteration $v$, that estimates $\widehat{\mathbf{x}}^{1} \ldots \widehat{\mathbf{x}}^{v-1}$ for the $N(v-1)$ first components of $\mathbf{x}$ have been obtained. One chooses here to evaluate the MAP estimate

$$
\widehat{\mathbf{x}}_{\mathrm{MAP}}^{v}=\arg \max _{\mathbf{x}^{v}} p_{X \mid R}\left(\mathbf{x}^{v} \mid \mathbf{r}_{1: M}^{v}, \widehat{\mathbf{x}}^{1} \ldots \widehat{\mathbf{x}}^{v-1}\right),
$$

of $\mathbf{x}^{v}$, using the knowledge of $\mathbf{r}_{1: M}^{v}$ and $\widehat{\mathbf{x}}^{1} \ldots \widehat{\mathbf{x}}^{v-1}$. Using Bayes' rule, one gets

$$
\begin{gathered}
\widehat{\mathbf{x}}_{\mathrm{MAP}}^{v}=\arg \max _{\mathbf{x}^{v}} p_{R \mid X}\left(\mathbf{r}_{1: M}^{v} \mid \mathbf{x}^{v}, \widehat{\mathbf{x}}^{1} \ldots \widehat{\mathbf{x}}^{v-1}\right) \\
p_{X}\left(\mathbf{x}^{v} \mid \widehat{\mathbf{x}}^{1} \ldots \widehat{\mathbf{x}}^{v-1}\right)
\end{gathered}
$$

where

$$
\begin{aligned}
p_{R \mid X} & \left(\mathbf{r}_{1: M}^{v} \mid \mathbf{x}^{v}, \widehat{\mathbf{x}}^{1} \ldots \widehat{\mathbf{x}}^{v-1}\right) \\
& =\sum_{\mathbf{z}_{1: M}} p_{R Z \mid X}\left(\mathbf{r}_{1: M}^{v}, \mathbf{z}_{1: M} \mid \mathbf{x}^{v}, \widehat{\mathbf{x}}^{1} \ldots \widehat{\mathbf{x}}^{v-1}\right) \\
& =\sum_{\mathbf{z}_{1: M}} p_{R \mid Z}\left(\mathbf{r}_{1: M}^{v} \mid \mathbf{z}_{1: M}\right) p_{Z \mid X}\left(\mathbf{z}_{1: M} \mid \mathbf{x}^{v}, \widehat{\mathbf{x}}^{1} \ldots \widehat{\mathbf{x}}^{v-1}\right) .
\end{aligned}
$$

Then

$$
\begin{aligned}
& p_{Z \mid X}\left(\mathbf{z}_{1: M} \mid \mathbf{x}^{v}, \widehat{\mathbf{x}}^{1} \ldots \widehat{\mathbf{x}}^{v-1}\right)= \\
& p_{Z \mid X}\left(\mathbf{z}_{1: M} \mid \mathbf{H}_{0} \mathbf{x}^{v}+\sum_{u=1}^{K-1} \mathbf{H}_{u} \widehat{\mathbf{x}}^{v-u}\right) .
\end{aligned}
$$

According to (2), one has 
Table 1. Values of $p\left(\mathbf{r}_{m} \mid z_{m}\right)$

\begin{tabular}{c|c|c|c|c}
$p\left(\mathbf{r}_{m} \mid z_{m}\right)$ & $z_{m}=0$ & $z_{m}=1$ & $z_{m}=2$ & $z_{m}=3$ \\
\hline$m=1$ & 0.1984 & 0.1335 & 0.3993 & 0.2688 \\
\hline$m=2$ & 0.0183 & 0.0010 & 0.9310 & 0.0497 \\
\hline$m=3$ & 0.0400 & 0.0260 & 0.5663 & 0.3678 \\
\hline$p_{Z \mid X}\left(\mathbf{z}_{1: M} \mid \mathbf{H}_{0} \mathbf{x}^{v}+\sum_{u=1}^{K-1} \mathbf{H}_{u} \widehat{\mathbf{x}}^{v-u}\right)$ \\
$=I_{\left[\mathbf{s}\left(\mathbf{z}_{1: M}\right)\right]}\left(\mathbf{H}_{0} \mathbf{x}^{v}+\sum_{u=1}^{K-1} \mathbf{H}_{u} \widehat{\mathbf{x}}^{v-u}\right)$, \\
$=I_{\left[\mathbf{s}^{\prime}\left(\mathbf{z}_{1: M}\right)\right]}\left(\mathbf{H}_{0} \mathbf{x}^{v}\right)$.
\end{tabular}

where

$$
\left[\mathbf{s}^{\prime}\left(\mathbf{z}_{1: M}\right)\right]=\left[\underline{\mathbf{s}}\left(\mathbf{z}_{1: M}\right)-\sum_{u=1}^{K-1} \mathbf{H}_{u} \widehat{\mathbf{x}}^{v-u}, \overline{\mathbf{s}}\left(\mathbf{z}_{1: M}\right)-\sum_{u=1}^{K-1} \mathbf{H}_{u} \widehat{\mathbf{x}}^{v-u}\right]
$$

One finally gets

$$
\begin{aligned}
\widehat{\mathbf{x}}_{\mathrm{MAP}}^{v} & =\arg \max _{\mathbf{x}^{v}} p_{X}\left(\mathbf{x}^{v} \mid \widehat{\mathbf{x}}^{1} \ldots \widehat{\mathbf{x}}^{v-1}\right) \\
& \sum_{\mathbf{z}_{1: M}} p_{R \mid Z}\left(\mathbf{r}_{1: M}^{v} \mid \mathbf{z}_{1: M}\right) I_{\left[\mathbf{s}^{\prime}\left(\mathbf{z}_{1: M}\right)\right]}\left(\mathbf{H}_{0} \mathbf{x}^{v}\right) .
\end{aligned}
$$

Using the same approximation for the sum over all possible $\mathbf{z}_{1: M}$ as in Section 4.2, one gets the approximate linearlyconstrained optimization problem derived from (28)

$$
\begin{aligned}
\widehat{\mathbf{x}}_{\mathrm{MAP}}^{v} & =\arg \max _{\mathbf{x}^{v}} p_{X}\left(\mathbf{x}^{v} \mid \widehat{\mathbf{x}}^{1} \ldots \widehat{\mathbf{x}}^{v-1}\right) \\
\text { st } & \mathbf{H}_{0} \mathbf{x}^{v} \leqslant \overline{\mathbf{s}}\left(\mathbf{z}_{1: M}^{*}\right)-\sum_{u=1}^{K-1} \mathbf{H}_{u} \widehat{\mathbf{x}}^{v-u}, \\
& -\mathbf{H}_{0} \mathbf{x}^{v} \leqslant-\underline{\mathbf{s}}\left(\mathbf{z}_{1: M}^{*}\right)+\sum_{u=1}^{K-1} \mathbf{H}_{u} \widehat{\mathbf{x}}^{v-u},
\end{aligned}
$$

where $\mathbf{z}_{1: M}^{*}$ is the value of $\mathbf{z}_{1: M}$ maximizing $p_{R \mid Z}\left(\mathbf{r}_{1: M}^{v} \mid \mathbf{z}_{1: M}\right)$. The estimation of $\mathbf{x}^{1}$ at initialization is performed as in Section 4.2, using $\mathbf{r}_{1: M}^{1}$ only and $\mathbf{H}=\mathbf{H}_{0}$.

\section{FIRST (TOY) EXAMPLE}

In the following example, $N=2, M=3$, and

$$
\mathbf{H}=\left(\begin{array}{cc}
1 & 0 \\
-1 / 2 & \sqrt{3} / 2 \\
-1 / 2 & -\sqrt{3} / 2
\end{array}\right) \text {. }
$$

A parity-check matrix associated to $\mathbf{H}$ is $\mathbf{P}=(1,1,1)$. Assume that $\mathbf{X} \sim \mathcal{N}\left(0, I_{2}\right)$ and that $\mathbf{x}=(-1.0279,0.1167)^{T}$ is placed at the input of $\mathbf{H}$ to get $\mathbf{y}=\mathbf{H x}=$ $(-1.0279,0.6150,0.4129)^{T}$. A 2-bit quantizer is considered with $\left.\left.\left[s_{0}\right]=\right]-\infty,-0.6852\right],\left[s_{1}\right]=[-0.6852,0.0000]$, $\left[s_{2}\right]=[0.0000,0.6852]$, and $\left[s_{3}\right]=[0.6852,+\infty[$. Then $\mathbf{z}=q(\mathbf{y})=(0,2,2)^{T}$ is transmitted over some noisy channel. From the channel output, assume that one obtains the values of $p\left(\mathbf{r}_{m} \mid z_{m}\right)$ reported in Table 1 . One may then determine whether there exists some feasible $\mathbf{x}$ for various combinations of $\mathbf{z}_{1: 3}$ sorted by decreasing value of

\begin{tabular}{|c|c|c|}
\hline $\mathbf{z}_{1: 3}$ & $p\left(\mathbf{r}_{1: 3} \mid \mathbf{z}_{1: 3}\right)$ & consistency test \\
\hline $\mathbf{z}^{(1)}=(2,2,2)$ & 0.2105 & $\mathbf{0} \notin \mathbf{P}\left[\mathbf{s}\left(\mathbf{z}^{(1)}\right)\right]$ \\
\hline $\mathbf{z}^{(2)}=(3,2,2)$ & 0.1417 & $\mathbf{0} \notin \mathbf{P}\left[\mathbf{s}\left(\mathbf{z}^{(2)}\right)\right]$ \\
\hline $\mathbf{z}^{(3)}=(2,2,3)$ & 0.1367 & $\mathbf{0} \notin \mathbf{P}\left[\mathbf{s}\left(\mathbf{z}^{(3)}\right)\right]$ \\
\hline $\mathbf{z}^{(4)}=(0,2,2)$ & 0.1046 & $\mathbf{0} \in \mathbf{P}\left[\mathbf{s}\left(\mathbf{z}^{(4)}\right)\right]$ \\
\hline $\mathbf{z}^{(5)}=(3,2,3)$ & 0.0920 & $\mathbf{0} \notin \mathbf{P}\left[\mathbf{s}\left(\mathbf{z}^{(5)}\right)\right]$ \\
\hline
\end{tabular}
$p\left(\mathbf{r}_{1: 3} \mid \mathbf{z}_{1: 3}\right)$, see Table 2 . The consistency test using interval analysis (20) is used for that purpose.

Finally, one selects $\mathbf{z}^{(4)}=(0,2,2)$ to solve (15) where instead of maximizing $p_{X}(\mathbf{x})$, one minimizes $\mathbf{x}^{\prime} \mathbf{x}$, since
Table 2. The 5 most likely $\mathbf{z}_{1: M}$ and the corresponding consistency test

$\mathbf{X} \sim \mathcal{N}\left(0, I_{2}\right)$. One thus gets a simple quadratic program with linear constraints. The solution is

$$
\widehat{\mathbf{x}}_{\mathrm{MAP}}=(-0.6853,0)^{T^{\prime}},
$$

which is reasonably close to $\mathbf{x}=(-1.0279,0.1167)^{T}$.

\section{SECOND EXAMPLE}

This section illustrates the recursive implementation of the MAP estimator of Section 4.4. The expansion is performed by an OFB performing simultaneously decorrelation of the samples of the input signal and redundancy introduction, due to the overcomplete representation provided by the output subbands, see Vaidyanathan [1993]. The estimation of the input signal of the OFB from noisy observation of its quantized subbands may be cast in the general estimation framework presented in Section 2, where the transform matrix $\mathbf{H}$ is as in (21). Some basic notions on OFBs are first recalled before illustrating the algorithm of Section 4.4.

Figure 2 represents an OFB consisting of $M$ analysis filters $\left\{h_{m}(n), n \in \mathbb{N}\right\}, m=0 \ldots M-1$, followed by a set of decimators with a decimation rate $N<M$.

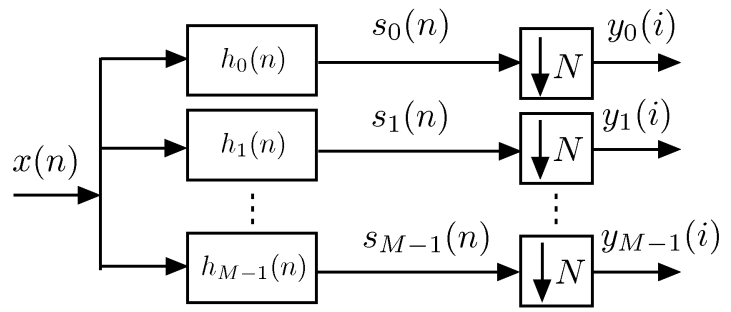

Fig. 2. Representation of an OFB

The input signal $x(n), n \in \mathbb{N}$, goes first through the analysis filters and $M$ output signals $\left\{s_{m}(n), n \in \mathbb{N}\right\}$, $m=0 \ldots M-1$ are obtained. The $n$-th sample of the $m$-th analysis filter output, $s_{m}(n)$ is expressed as

$$
s_{m}(n)=\sum_{k=-\infty}^{+\infty} h_{m}(k) x(n-k)
$$

When the analysis filters have finite impulse responses (FIR) and are of maximal length $L,(30)$ becomes

$$
s_{m}(n)=\sum_{k=1}^{L} h_{m}(k) x(n-k) .
$$

After downsampling with a decimation factor $N<M$, one obtains the downsampled signals

$$
y_{m}(i)=\sum_{k=1}^{I_{e}} h_{m}(k) x(i N-k) \text {. }
$$




\subsection{Polyphase representation}

One can see from (32) that the computation of the terms $s_{m}(n)$ is not necessary when $n$ is not a multiple of $N$. The polyphase representation of the OFB in which the downsampling operation is performed before the filtering can then be considered, see Figure 3.

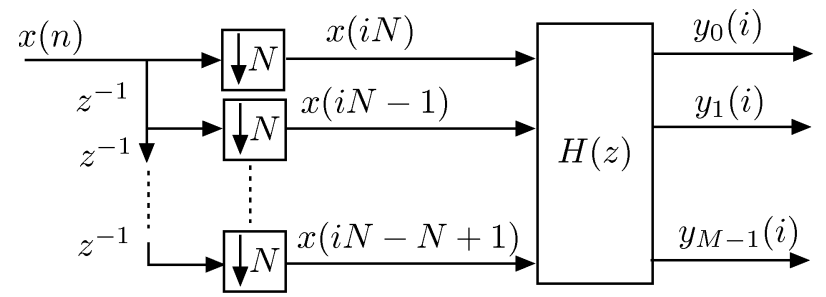

Fig. 3. Polyphase representation of an OFB

Without loss of generality, one can take $L$ as the smallest multiple of $N$ that is greater than the largest length of the subband impulse response (zeros may be added to impulse responses if necessary). Then $L=N \times K$ for some positive integer $K$. Introducing the matrices

$$
\mathbf{H}_{k}=\left(\begin{array}{ccc}
h_{0}(k N) & \cdots & h_{0}((k+1) N-1) \\
\vdots & \vdots & \vdots \\
h_{M-1}(k N) & \cdots & h_{M-1}((k+1) N-1)
\end{array}\right)
$$

where $k=0 \ldots K-1$, the temporal relation between the input and the output of the OFB can be written as in (22) where for $v=1 \ldots V$

$$
\mathbf{x}^{v}=(x((v-1) N+1) \ldots x(v N))^{T},
$$

and

$$
\mathbf{y}^{v}=(y((v-1) M+1) \ldots y(v M))^{T} .
$$

One may also introduce the polyphase matrix of the considered OFB given by

$$
\mathbf{H}(z)=\sum_{k=0}^{K-1} \mathbf{H}_{k} z^{-r} .
$$

\subsection{Parity-check matrix}

In order to enable perfect reconstruction the columns of $\mathbf{H}(z)$ must be of rank $N$. In this case $\mathbf{H}(z)$ admits a SmithMcMillan decomposition

$$
\mathbf{H}(z)=\mathbf{U}(z)\left(\begin{array}{c}
\boldsymbol{\Lambda}(z) \\
\mathbf{0}
\end{array}\right) \mathbf{W}(z)
$$

where $\mathbf{U}(z)$ and $\mathbf{W}(z)$ are invertible matrices of size $M \times$ $M$ and $N \times N$ respectively and $\boldsymbol{\Lambda}(z)$ is a $N \times N$ diagonal matrix Labeau et al. [2005]. One can put the inverse of $\mathbf{U}(z)$ in the following form

$$
\mathbf{U}^{-1}(z)=\left(\begin{array}{l}
\mathbf{V}(z) \\
\mathbf{P}(z)
\end{array}\right)
$$

where $\mathbf{V}(z)$ and $\mathbf{P}(z)$ are matrices of size $N \times M$ and $(M-N) \times M$ respectively and the rows of $\mathbf{P}(z)$ are of rank $M-N$. The matrix $\mathbf{P}(z)$ is the parity-check polyphase matrix associated to $\mathbf{H}(z)$ and it verifies

$$
\mathbf{P}(z) \mathbf{H}(z)=\mathbf{0}, \forall z \in \mathbb{C}
$$

When $\mathbf{H}(z)$ is the polyphase matrix of an FIR filterbank, the parity check matrix $\mathbf{P}(z)$ is also associated to an FIR filterbank and can be written as follows:

$$
\mathbf{P}(z)=\sum_{k=0}^{K^{\prime}-1} \mathbf{P}_{k} z^{-k}
$$

where $\mathbf{P}_{k}, k=0 \ldots K^{\prime}$ are $(M-N) \times M$ matrices. Writing (35) in the temporal domain for each output $\mathbf{y}^{v}$ of the OFB leads to

$$
\sum_{k=0}^{K^{\prime}-1} \mathbf{P}_{k} \mathbf{y}^{v-k}=\mathbf{0}
$$

where $\mathbf{y}^{v}=0$, if $v \leqslant 0$. The relation (37) defines a set of $M-N$ equations, which may be used to check whether a subband signal could have been generated by the OFB. They are referred to as the parity-check test (PCT) equations.

\subsection{Quantization and transmission}

Each component $y_{m}^{v}, m=1 \ldots M$, of the vector $\mathbf{y}^{v}$, $v=1 \ldots V$, is quantized using a scalar quantizer $q_{m}$ of step $\delta_{m}$ and of rate $\rho_{m}$. The quantization intervals defined in (2) are then expressed as follows

$$
\begin{aligned}
& \left.\left.\left[s_{0}\right]=\right]-\infty, q_{m}^{-1}(0)+\delta_{m} / 2\right], \\
& {\left[s_{z}\right]=\left[q_{m}^{-1}(z)-\delta_{m} / 2, q_{m}^{-1}(z)+\delta_{m} / 2\right], z=1 \ldots 2^{\rho_{m}}-2} \\
& {\left[s_{2^{\rho_{m}-1}}\right]=\left[q_{m}^{-1}\left(2^{\rho_{m}}-1\right)-\delta_{m} / 2,+\infty[,\right.}
\end{aligned}
$$

where $q_{m}^{-1}$ is the inverse quantizer associated to $q_{m}$. The resulting quantization indexes are then binarized. The binarization of each $z_{m}$ provides a sequence $b\left(z_{m}\right)$ of $\rho_{m}$ bits that is modulated using a BPSK modulation $\mu$ to get a sequence $\mu\left(b\left(z_{m}\right)\right)=\left\{\mu_{m}^{0} \ldots \mu_{m}^{\rho_{m}-1}\right\} \in\{-1,1\}^{\rho_{m}}$. The sequence $\mu\left(b\left(z_{m}\right)\right)$ is then transmitted over a memoryless channel characterized by its transition probability $g(r \mid b)$. At the channel output the vector $\mathbf{r}_{m}=\left(r_{m}^{0} \ldots r_{m}^{\rho_{m}}-1\right)^{T}$ of real or complex values is obtained. Using the fact that $b$ and $\mu$ are deterministic and that the channel is memoryless, the likelihood of a particular quantization index $z_{m}$ is evaluated as

$$
\begin{aligned}
p_{R \mid Z}\left(\mathbf{r}_{m} \mid z_{m}\right) & =p_{R \mid Z}\left(\mathbf{r}_{m} \mid \mu\left(b\left(z_{m}\right)\right)\right) \\
& =\prod_{i=0}^{\rho_{m}-1} g\left(r_{m}^{i} \mid b_{m}^{i}\right) .
\end{aligned}
$$

\subsection{Estimation algorithm}

The estimation of the input $\mathbf{x}$ can be operated using the iterative algorithm of Section 4.4. A possible implementation is as follows.

(1) Sort the $N^{\prime}$ best values of $\mathbf{z}_{1: M}$ in terms of $p_{R \mid Z}\left(\mathbf{r}_{m}^{v} \mid \mathbf{z}_{1: M}\right)$ and store them in $\mathcal{L}=\left\{\mathbf{z}_{1: M}^{(0)} \ldots \mathbf{z}_{1: M}^{\left(N^{\prime}-1\right)}\right\}$

(2) $k=0$;

(3) Do
(a) If $\mathbf{0} \in \mathbf{P}_{0}\left[\mathbf{s}\left(\mathbf{z}_{1: M}^{(k)}\right)\right]+\sum_{u=1}^{K^{\prime}-1} \mathbf{P}_{u} \widehat{\mathbf{x}}^{\nu-u}$
(b) Solve (29)
(c) If a feasible solution $\widehat{\mathbf{x}}^{v}$ is found, return $\widehat{\mathbf{x}}^{v}$;
(d) Else $k=k+1$;

(4) While $k \leq N^{\prime}$.

(5) Return error.

Note that Step 3(a) involves basic operations on intervals. 


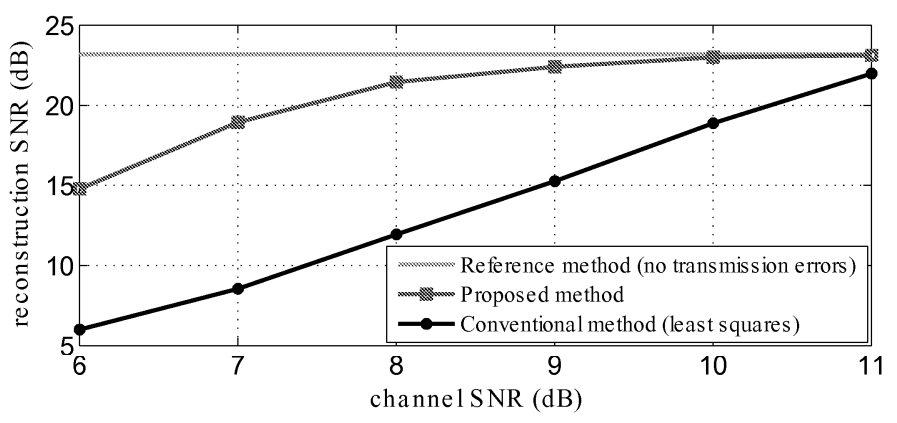

Fig. 4. SNR of the reconstructed signal as a function of the channel SNR for the 4 lines of lena.pgm

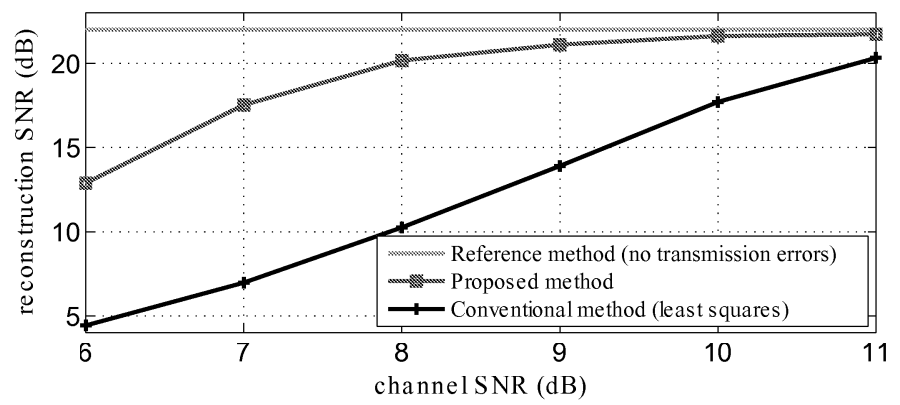

Fig. 5. SNR of the reconstructed signal as a function of the channel SNR for a continuous-valued one-dimensional signal

\subsection{Experimental results}

Consider an OFB with $M=6$ and $N=4$ with $\mathbf{H}$ defined as in (21) with $K=2$ and

$$
\mathbf{H}_{0}=\left(\begin{array}{cccc}
1 & 1 & 0 & 0 \\
0 & 0 & 0 & 0 \\
-1 & 1 & 0 & 0 \\
1 & 0 & 0 & 0 \\
0 & 0 & 0 & 0 \\
0 & 0 & 0 & 0
\end{array}\right), \mathbf{H}_{1}=\left(\begin{array}{cccc}
0 & 0 & 0 & 0 \\
0 & 0 & 1 & 1 \\
0 & 0 & 0 & 0 \\
0 & 0 & 0 & 1 \\
0 & 1 & 1 & 0 \\
0 & -1 & 1 & 0
\end{array}\right)
$$

Two types of signals have been considered: a discretevalued signal formed by 4 lines of Lena.pgm and a continuous-valued one corresponding to the realizations of a zero-mean unit variance correlated Gaussian noise with correlation coefficient $c=0.9$. The first $N V=2000$ samples have been kept for each signal, $V=500$. The output vector $\mathbf{y}$ is then of length $M V=3000$. The $m$ th component of the vector $\mathbf{y}^{v}, v=1 \ldots V$, is quantized with a rate $\rho_{m}=4$ for $m \in\{1,2,4,5\}$ and $\rho_{m}=2$ for $m \in\{3,6\}$. A BPSK modulation of the resulting quantized indexes has been performed before their transmission over an AWGN channel with SNR level between $6 \mathrm{~dB}$ and $11 \mathrm{~dB}$. The estimation algorithm presented in Section 6.4 has been used with $N^{\prime}=20$. The results have been averaged over 250 noise realizations for both signals.

Figures 4 and 5 show the average SNR of the reconstructed signal as a function of the channel SNR. The noiseless signal reconstructed after inverse quantization (in green) serves as reference. The signal estimated using the algorithm presented in Section 6.4 (in red) has a higher SNR than the one estimated using the value of the quantization indexes $\mathbf{z}_{1: M}$ maximizing $p_{R \mid Z}\left(\mathbf{r}_{1: M}^{v} \mid \mathbf{z}_{1: M}\right), v=1 \ldots V$ (in black).

\section{CONCLUSIONS}

This paper presents a MAP estimator for some vector $\mathbf{x}$ from its quantized and noisy linear measurements. The complexity of the optimal MAP estimator is intractable in general. Two suboptimal solutions have been proposed, one of which being iterative to be able to handle large-scale problems. Leveraging on techniques from interval analysis, it is possible to quickly eliminate solutions which are not consistent with the signal model, and the quantization noise.

These techniques have been applied to the estimation of the input signal of an OFB using noisy measurements of its quantized subbands. The experimental results show that when the channel is noisy, this approach performs better in terms of reconstruction SNR than classical least-squares reconstruction.

\section{REFERENCES}

M. Abid, M. Kieffer, and B. Pesquet-Popescu. Consistent reconstruction of the input of an oversampled filter bank from noisy subbands. In European Signal Processing Conference, EUSIPCO, Barcelone, Spain, 2011.

M. Akbari and F. Labeau. Recovering the output of an OFB in the case of instantaneous erasures in sub-band domain. In Proc. MMSP, pages 274-279, St Malo, France, 2010.

J. B. Anderson. Digital Transmission Engineering. Wiley, second edition, 2005.

D.L. Donoho, A.Maleki, and A. Montanari. Message passing algorithm for compressed sensing. arXiv:0907.3574v1 [cs.IT], 2009.

V. K. Goyal. Single and Multiple Description Transform Coding with Bases and Frames. SIAM, Philadelphia, PA, 2002.

V. K Goyal, J. Kovacevic, and J.A. Kelner. Quantized frame expansions with erasures. Journal of Appl. and Comput. Harmonic Analysis, 10(3):203-233, 2001.

L. Jaulin, M. Kieffer, O. Didrit, and E. Walter. Applied Interval Analysis. Springer-Verlag, London, 2001.

J. Kovacević, P. L. Dragotti, and V. K Goyal. Filter bank frame expansions with erasures. IEEE trans. Information Theory, 48 (6):1439-1450, 2002.

F. R. Kschischang, B. J. Frey, and H. A. Loeliger. Factor graphs and the sum-product algorithm. IEEE Transactions on information theory, 47(2):498-519, 2001.

F. Labeau, J.C. Chiang, M. Kieffer, P. Duhamel, L. Vandendorpe, and B. Mack. Oversampled filter banks as error correcting codes: theory and impulse correction. IEEE trans. on Signal Processing, 53(12):4619 - 4630, 2005

S. Rangan. Estimation with Random Linear Mixing, Belief Propagation and Compressed Sensing. 44th Annual Conference on Information Sciences and Systems (CISS), pages 1-6, 2010.

S. Rangan. Generalized approximate message passing for estiamation with random linear mixing. IEEE International Symposium on Information Theory Proceedings (ISIT), pages 2168-2172, 2011.

G. Rath and C. Guillemot. Frame-theoretic analysis of DFT codes with erasures. IEEE trans. on Signal Processing, 52(2):447-460, 2004

G. R. Redinbo. Decoding real block codes: Activity detection, wiener estimation. IEEE trans. on Information Theory, 46(2):609-623, 2000 .

P. P. Vaidyanathan. Multirate Systems and Filterbanks. PrenticeHall, Englewood-Cliffs, NJ, 1993. 